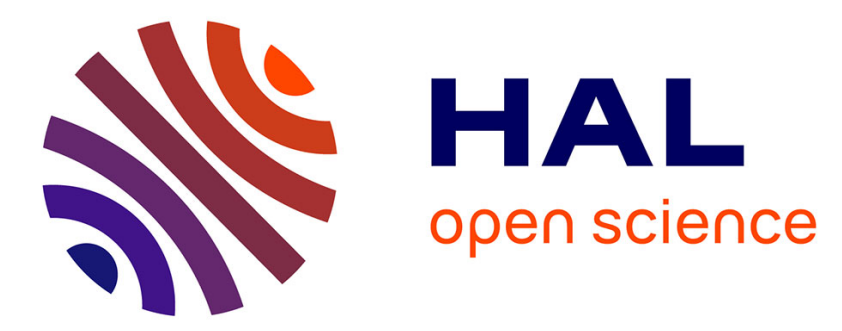

\title{
Optical and Near-Infrared Spectroscopy of the Black Hole Swift J1753.5-0127
}

Farid Rahoui, John A. Tomsick, Mickael Coriat, Stéphane Corbel, Felix Fürst, Poshak Gandhi, Simone Migliari, Emrah Kalemci, Daniel Stern, Anastasios K.

Tzioumis

\section{To cite this version:}

Farid Rahoui, John A. Tomsick, Mickael Coriat, Stéphane Corbel, Felix Fürst, et al.. Optical and Near-Infrared Spectroscopy of the Black Hole Swift J1753.5-0127. The Astrophysical journal letters, 2015, 810 (2), pp.161. 10.1088/0004-637X/810/2/161 . insu-01343963

\section{HAL Id: insu-01343963 https://hal-insu.archives-ouvertes.fr/insu-01343963}

Submitted on 18 May 2017

HAL is a multi-disciplinary open access archive for the deposit and dissemination of scientific research documents, whether they are published or not. The documents may come from teaching and research institutions in France or abroad, or from public or private research centers.
L'archive ouverte pluridisciplinaire HAL, est destinée au dépôt et à la diffusion de documents scientifiques de niveau recherche, publiés ou non, émanant des établissements d'enseignement et de recherche français ou étrangers, des laboratoires publics ou privés. 


\title{
OPTICAL AND NEAR-INFRARED SPECTROSCOPY OF THE BLACK HOLE SWIFT J1753.5-0127
}

\author{
Farid Rahoui $^{1,2}$, John A. Tomsick ${ }^{3}$, Mickael Coriat ${ }^{4,5}$, Stéphane Corbel $^{6,7}$, Felix Fürst $^{8}$, Poshak Gandhi $^{9}$, \\ Emrah Kalemci $^{10}$, Simone Migliari ${ }^{11}$, Daniel Stern ${ }^{12}$, and Anastasios K. Tzioumis ${ }^{13}$ \\ ${ }^{1}$ European Southern Observatory, K. Schwarzschild-Strasse 2, D-85748 Garching bei München, Germany; frahoui@eso.org \\ ${ }^{2}$ Department of Astronomy, Harvard University, 60 Garden street, Cambridge, MA 02138, USA \\ ${ }^{3}$ Space Sciences Laboratory, 7 Gauss Way, University of California, Berkeley, CA 94720-7450, USA \\ ${ }^{4}$ IRAP, Université de Toulouse, UPS, 9 Avenue du colonel Roche, F-31028 Toulouse Cedex 4, France \\ ${ }^{5}$ CNRS, UMR5277, F-31028 Toulouse, France \\ ${ }^{6}$ Laboratoire AIM (CEA/IRFU—CNRS/INSU—Université Paris Diderot), CEA DSM/IRFU/SAp, F-91191 Gif-sur-Yvette, France \\ ${ }^{7}$ Station de Radioastronomie de Nanay, Observatoire de Paris, PSL Research University, CNRS, Univ. Orlans, OSUC, F-18330 Nanay, France \\ ${ }^{8}$ California Institute of Technology, 1200 East California Boulevard, Pasadena, CA 91125, USA \\ ${ }^{9}$ Department of Physics, University of Durham, South Road, Durham DH1 3LE, UK \\ ${ }^{10}$ Sabanci University, Orhanli-Tuzla, Istanbul, 34956, Turkey \\ ${ }^{11}$ European Space Astronomy Centre, Apartado/P.O. Box 78, Villanueva de la Canada, E-28691 Madrid, Spain \\ 12 Jet Propulsion Laboratory, California Institute of Technology, 4800 Oak Grove Drive, Pasadena, CA 91109, USA \\ ${ }^{13}$ CSIRO Astronomy and Space Science, Australia Telescope National Facility, P.O. Box 76, Epping, NSW 1710, Australia \\ Received 2015 May 9; accepted 2015 August 3; published 2015 September 10
}

\begin{abstract}
We report on a multiwavelength observational campaign of the black hole (BH) X-ray binary Swift J1753.5-0127 that consists of an ESO/X-shooter spectrum supported by contemporaneous Swift/X-ray Telescope+Ultra-Violet/Optical Telescope (UVOT) and Australia Telescope Compact Array data. Interstellar medium absorption lines in the X-shooter spectrum allow us to determine $E(B-V)=0.45 \pm 0.02$ along the line of sight to the source. We also report detection of emission signatures of $\mathrm{He}$ II $\lambda 4686, \mathrm{H} \alpha$, and, for the first time, $\mathrm{H}_{\mathrm{I}} \lambda 10906$ and $\mathrm{Pa} \beta$. The double-peaked morphology of these four lines is typical of the chromosphere of a rotating accretion disk. Nonetheless, the paucity of disk features points toward a low level of irradiation in the system. This is confirmed through spectral energy distribution modeling, and we find that the UVOT $+\mathrm{X}$-shooter continuum mostly stems from the thermal emission of a viscous disk. We speculate that the absence of reprocessing is due to the compactness of an illumination-induced envelope that fails to reflect enough incoming hard X-ray photons back to the outer regions. The disk also marginally contributes to the Compton-dominated $\mathrm{X}$-ray emission and is strongly truncated, with an inner radius about 1000 times larger than the BH's gravitational radius. A near-infrared excess is present, and we associate it with synchrotron radiation from a compact jet. However, the measured X-ray flux is significantly higher than what can be explained by the optically thin synchrotron jet component. We discuss these findings in the framework of the radio-quiet versus X-ray-bright hypothesis, favoring the presence of a residual disk, predicted by evaporation models, that contributes to the X-ray emission without enhancing the radio flux.
\end{abstract}

Key words: accretion, accretion disks - binaries: close - infrared: stars - ISM: jets and outflows - stars: individual (Swift J1753.5-0127) - X-rays: binaries

\section{INTRODUCTION}

Microquasars are disk-accreting X-ray binaries characterized by the presence of collimated bipolar radio ejections called jets. When in outburst, they transition between two canonical spectral states, dubbed soft when the disk dominates the X-ray emission and hard when continuous compact radio jets are present (see, e.g., Fender et al. 2004). In the hard state, microquasars have consequently long been known to exhibit a very tight radio/X-ray correlation spanning several orders of magnitude. First measured for a couple of sources almost 20 years ago (Hannikainen et al. 1998; Corbel et al. 2000), this relation was progressively extended to almost all known systems (see, e.g., Gallo et al. 2003; Corbel et al. 2013; Gallo et al. 2014). Nonetheless, it quickly appeared that what was initially thought to be a universal relation, $F_{\mathrm{R}} \propto F_{\mathrm{X}}^{0.6-0.7}$, is actually not followed by a growing number of outliers that exhibit a lower-than-expected radio flux for a given X-ray luminosity (see, e.g., Corbel et al. 2004; Gallo 2007; Coriat et al. 2011). Such sources are often referred to as radio-quiet microquasars (Coriat et al. 2011; Soleri \& Fender 2011), by analogy with active galactic nuclei.
One such radio-quiet microquasar, Swift J1753.5-0127, was discovered by the Swift Burst Alert Telescope on 2005 June 30 (Palmer et al. 2005). It is the microquasar with the shortest orbital period, first found to be lower than $3.25 \mathrm{hr}$ (Zurita et al. 2008), and later refined to $2.85 \mathrm{hr}$ (Neustroev et al. 2014). With such a short period, it is very likely that the companion star is a cold $\mathrm{M}$ dwarf, although it has never been formally identified. In fact, besides the orbit, very little is known about the parameters of the system. The presence of non-P-Cygni emission lines in its ultraviolet (UV) spectrum and the absence of eclipse put the inclination in the range of $40^{\circ}-80^{\circ}$ (Froning et al. 2014), and Reis et al. (2009) measured $i=55_{-7}^{\circ+2}$ from $\mathrm{X}$-ray spectral fitting. The distance is also poorly constrained but is believed to be between 1 and $8 \mathrm{kpc}$ (Cadolle Bel et al. 2007; Durant et al. 2008), although larger values cannot be ruled out. Likewise, the black hole $(\mathrm{BH})$ mass is unknown, with estimates ranging from a typical $M_{\mathrm{BH}} \sim 10 M_{\odot}$ value (Cadolle Bel et al. 2007; Zurita et al. 2008) to $M_{\mathrm{BH}} \leqslant 5 M_{\odot}$ (Neustroev et al. 2014).

Swift J1753.5-0127 has never returned to quiescence since the beginning of its outburst in 2005, and it remained almost 10 
Table 1

Summary of the Observations of Swift J1753.5-0127 We Made Use of in This Study

\begin{tabular}{|c|c|c|c|c|c|c|c|c|c|c|c|c|}
\hline Parameter & Swift/XRT & \multicolumn{6}{|c|}{ Swift/UVOT } & \multicolumn{3}{|c|}{ X-shooter } & \multicolumn{2}{|c|}{ ATCA } \\
\hline Date $^{a}$ & 56885.3 & \multicolumn{6}{|c|}{56885.3} & \multicolumn{3}{|c|}{56886.0} & \multicolumn{2}{|c|}{56885.3} \\
\hline Band $^{\mathrm{b}}$ & $0.4-7.5 \mathrm{keV}$ & $u w 2$ & um2 & $u w 1$ & $u$ & $b$ & $v$ & UVB & VIS & NIR & $5.5 \mathrm{GHz}$ & $9 \mathrm{Gl}$ \\
\hline Exposure $^{c}$ & 1920 & 628 & 454 & 304 & 156 & 157 & 156 & & 6720 & & \multicolumn{2}{|c|}{6060} \\
\hline
\end{tabular}

Notes.

a Starting date of the observation, in MJD.

b Energy band, filters, or central frequencies.

c Total exposure time on-source, in seconds.

years in the hard state before transitioning to the soft state in 2015 February. Although this extended period of heightened flux has put the source under a thorough scrutiny, the reasons behind this behavior remain uncertain. Moreover, whether the accretion disk is truncated as expected in the framework of advection-dominated accretion flows (ADAFs; see, e.g., Narayan \& Yi 1995) or extends up to its innermost stable circular orbit (ISCO) is still unclear. Recently, Froning et al. (2014) modeled the UV to near-infrared (NIR) spectral energy distribution (SED) of the source with a viscous accretion disk and concluded that the disk had to be strongly truncated. On the other hand, Reis et al. (2009) fitted the XMM-Newton spectrum with a reflection model and found no truncation at all. The compact jet contribution to the optical and NIR domains is also unknown.

In this paper we report on a multiwavelength study of Swift J1753.5-0127 in the hard state, centered on its optical and NIR spectroscopic emission as observed in 2014 August. Our main goals are (1) to study the geometry of the system and (2) to constrain the contribution of the compact jet to the optical and NIR emission. A work focusing on its early 2014 April radio and X-ray properties, when the source was in a low-luminosity hard state, is presented in a companion paper (Tomsick et al. 2015, hereafter T15). The observations and data reduction procedures are presented in Section 2. Section 3 is devoted to analysis of the optical and NIR spectrum, and Section 4 focuses on modeling the radio to X-ray SED. We discuss our results and their implications on our understanding of outliers to the radio/X-ray correlation of microquasars in Section 5, and we conclude in Section 6.

\section{OBSERVATIONS}

The data set consists of contemporaneous observations obtained on 2014 August 16 with (1) ESO+VLT/X-shooter (Obs. ID 093.D-0786, PI Rahoui); (2) the X-ray Telescope (XRT; Burrows et al. 2005) and the Ultra-Violet/Optical Telescope (UVOT, Roming et al. 2005) mounted on Swift (Gehrels et al. 2004) satellite (Obs. ID 00033140032, PI Tomsick); and (3) the Australia Telescope Compact Array (ATCA) at 5.5 and $9 \mathrm{GHz}$ (PI Coriat). A summary is given in Table 1.

\subsection{X-shooter Observations}

We obtained medium-resolution spectroscopy of Swift J1753.5-0127 with the three available arms of X-shooter (Vernet et al. 2011), UVB, VIS, and NIR (1".3, $1^{\prime \prime} .2$, and $1^{\prime \prime} .2$ slit-widths, respectively), giving simultaneous 300-2480 nm spectral coverage. Atmospheric conditions were medium to good, with a clear sky, seeing at $500 \mathrm{~nm}$ in the range $1^{\prime \prime} .2-1^{\prime \prime} .5$, and an airmass between 1.1 and 1.3. The exposure time of each individual frame was set to $210 \mathrm{~s}, 210 \mathrm{~s}$, and $70 \mathrm{~s}$ in the UVB, VIS, and NIR arms, respectively, and a total of 32, 32, and 96 frames were taken, with three NIR exposures being obtained for each UV/optical exposure. Standard ABBA dithering was used for effective background subtraction, and the A0V telluric standard star HR 6572 was observed in similar conditions for telluric feature removal and flux-calibration.

We reduced the data using the dedicated pipeline (v. 2.5.2) implemented in the ESO data reduction environment Reflex v. 2.6 (Freudling et al. 2013). It follows the standard steps for echelle spectroscopy reduction and produces a cleaned, background-subtracted, and wavelength-calibrated 2D spectroscopic image. We then used the routines apall and telluric implemented in IRAF v. $2 \cdot 16^{14}$ to extract the $1 \mathrm{D}$ spectra and remove the telluric features. We finally performed the flux calibration following the procedure presented in Rahoui et al. $(2012,2014)$ and rebinned the spectra from their original pixel resolution, i.e., $0.2 \AA, 0.2 \AA$, and $0.6 \AA$, to $1 \AA$, $1 \AA$, and $3 \AA$ in the UVB, VIS, and NIR arms, respectively.

\subsection{Swift Observations}

We reduced the XRT data with HEASOFT v. 6.16 and the 2014 October 2 calibration database version. We used xrtpipeline v. 0.13.1 to collect events in Windowed Timing mode to avoid pileup. The source and background spectra were extracted with xselect v. 2.4c using 40-pixel square boxes in the range $0.4-10 \mathrm{keV}$. We generated the ancillary response file with xrtmkarf and used the latest version (v. 015) of the response matrices provided by the Swift team. We rebinned the spectrum to obtain a minimum of 100 counts per channel.

The UVOT photometry was obtained in all filters, $u v w 2$, $u v m 2, u v w 1, u, b$, and $v$, and we produced an image in each of them with uvotimsum. We then used uvotsource to extract the source in a $5^{\prime \prime}$ region and the background counts in a $15^{\prime \prime}$ source-free circular aperture, respectively. The derived Swift J1753.5-0127 flux densities are listed in Table 2.

\subsection{ATCA Observations}

The array was in the compact H75 configuration, the observation was conducted with the Compact Array Broadband Backend (Wilson et al. 2011), and each frequency band was composed of $20481 \mathrm{MHz}$ channels. We used PKS B1934-638

\footnotetext{
14 IRAF is distributed by the National Optical Astronomy Observatories, which are operated by the Association of Universities for Research in Astronomy, Inc., under cooperative agreement with the National Science Foundation.
} 
Table 2

Swift J1753.5-0127 UVOT Flux Densities

\begin{tabular}{lcc}
\hline \hline Filter $^{\mathrm{a}}$ & $\lambda_{\mathrm{c}}{ }^{\mathrm{b}}$ & $F_{\lambda}^{\mathrm{c}}$ \\
\hline$u v w 2$ & 1928 & $8.17 \pm 0.39$ \\
$u m 2$ & 2246 & $6.05 \pm 0.38$ \\
$u w 1$ & 2600 & $8.55 \pm 0.49$ \\
$u$ & 3465 & $10.8 \pm 0.5$ \\
$b$ & 4392 & $9.27 \pm 0.42$ \\
$v$ & 5468 & $7.81 \pm 0.52$ \\
\hline
\end{tabular}

Notes.

${ }^{\text {a }}$ Filter designation

${ }^{\mathrm{b}}$ Central wavelength in $\AA$.

${ }^{c}$ Flux density in units of $10^{-16} \mathrm{erg} \mathrm{cm}^{-2} \mathrm{~s}^{-1} \AA^{-1}$.

for absolute flux and bandpass calibration, and PKS J1741-038 to calibrate the antenna gains as a function of time. Flagging, calibration, and imaging were carried out with the Multichannel Image Reconstruction, Image Analysis, and Display software (Sault et al. 1995). Owing to poor weather conditions, most of the observation was flagged and Swift J1753.5-0127 was not detected. We obtained the following $3 \sigma$ upper limits: $0.15 \mathrm{mJy}$ at $5.5 \mathrm{GHz}$ and $0.20 \mathrm{mJy}$ at $9 \mathrm{GHz}$, respectively.

\subsection{Wide-field Infrared Survey Explorer (WISE) Observations}

For comparison only, we also make use of WISE (Wright et al. 2010) data. The source was observed with WISE at five different epochs, around MJD 55,274, MJD 55,454, MJD 55,456, MJD 56,736, and MJD 56,918 (see Table 3 for the weight-averaged flux densities). In particular, the MJD 56,918 observation occurred about 1 month after our X-shooter observations (MJD 56,885) and consists of $13 \mathrm{~W} 1(3.35 \mu \mathrm{m})$ and W2 $(4.5 \mu \mathrm{m})$ frames of $7.7 \mathrm{~s}$ each obtained during the NEOWISE reactivation survey (Mainzer et al. 2014) between MJD 56,918.2 and MJD 56,919.1 (Figure 1). These magnitudes cannot therefore be considered as contemporaneous to our data, and this is the reason why we only superimpose the resulting weight-averaged flux densities on the modeled SEDs.

\section{THE OPTICAL AND NIR SPECTRUM}

Figure 2 displays the flux-calibrated X-shooter 350-2400 nm spectrum, on which the detected spectroscopic features are marked and the UVOT flux densities superimposed. Figure 3 highlights the lines-fitted with one or two Gaussian(s) - and Table 4 lists their main parameters. The FWHMs were quadratically corrected for the instrumental broadening, and the underlying continuum was locally assessed with a firstorder polynomial. The continuum level being the primary source of inaccuracy, each measurement was repeated several times with different continuum placements within the same wavelength range to obtain a set of values that eventually averaged out. The listed uncertainties are therefore the scatter to the mean rather than just statistical.

\subsection{The Spectroscopic Content}

In the optical domain, Neustroev et al. (2014) detected He II $\lambda 4686$ and $\mathrm{H} \alpha$ in 2013 August spectra. This was the first report of optical emission features since the very early outburst of Swift J1753.5-0127 (Torres et al. 2005), as both Cadolle Bel et al. (2007) and Durant et al. (2009) reported the absence of optical
Table 3

Swift J1753.5-0127 WISE Flux Densities

\begin{tabular}{lcc}
\hline \hline Observation Date & $F_{\mathrm{W} 1}{ }^{\mathrm{a}}$ & $F_{\mathrm{W} 2}{ }^{\mathrm{b}}$ \\
\hline MJD 55,274.4 & $0.63 \pm 0.01$ & $0.53 \pm 0.02$ \\
MJD 55,454.0 & $0.53 \pm 0.02$ & $0.44 \pm 0.02$ \\
MJD 55,456.5 & $0.53 \pm 0.02$ & $0.44 \pm 0.03$ \\
MJD 55,736.4 & $0.51 \pm 0.01$ & $0.49 \pm 0.02$ \\
MJD 55,918.7 & $0.51 \pm 0.01$ & $0.43 \pm 0.02$ \\
\hline
\end{tabular}

Notes.

${ }^{\mathrm{a}} \mathrm{W} 1$ flux density in mJy.

${ }^{\mathrm{b}}$ W2 flux density in mJy.
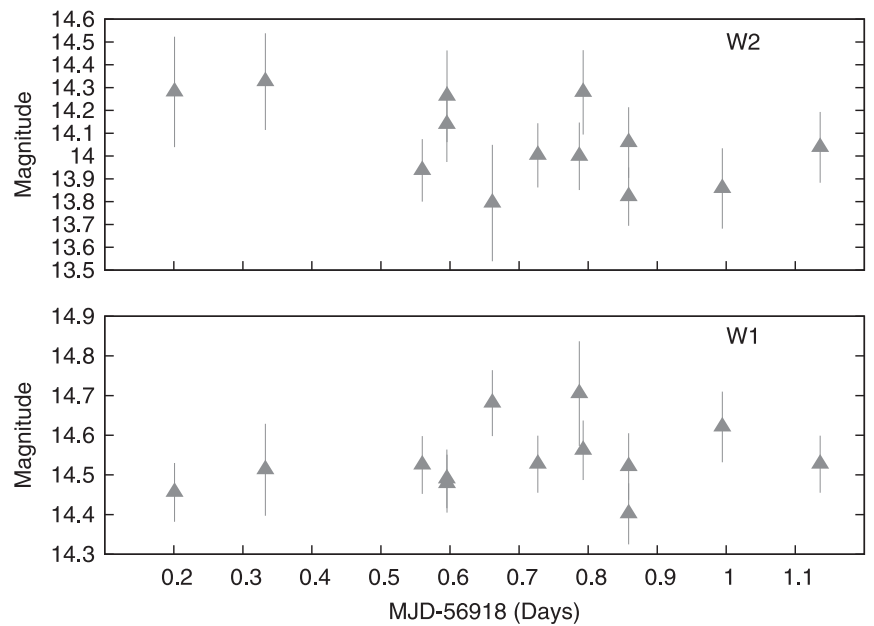

Figure 1. Swift J1753.5-0127 WISE magnitudes in W1 (3.35 $\mu \mathrm{m}$, bottom) and W2 $(4.6 \mu \mathrm{m}$, top) obtained about 1 month after our observations at 13 different epochs. No variability is present at $2 \sigma$.

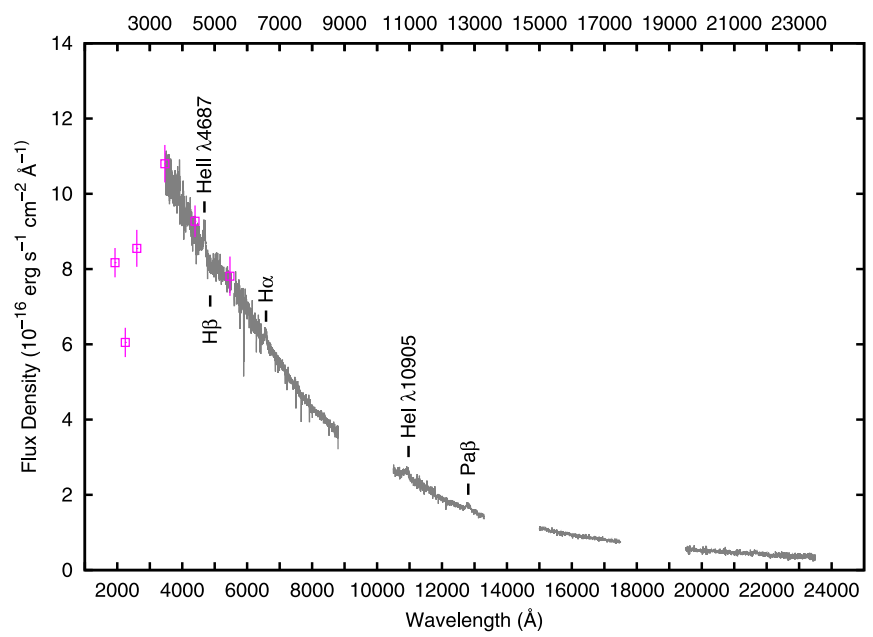

Figure 2. Flux-calibrated X-shooter spectrum of Swift J1753.5-0127, not corrected for the interstellar medium (ISM) extinction along the line of sight of the source. The detected spectroscopic lines are marked and the quasisimultaneous UVOT flux densities superimposed (magenta).

emission lines during observations that took place in 2005 August and 2007 June, respectively. Froning et al. (2014) also reported the detection of UV emission lines in 2012 October $H S T$ spectra. This likely hints at a renewed activity of the accretion disk anywhere between 2007 and 2012, although we cannot be more accurate. Neustroev et al. (2014) showed that the two lines were double peaked, and the authors measured total 

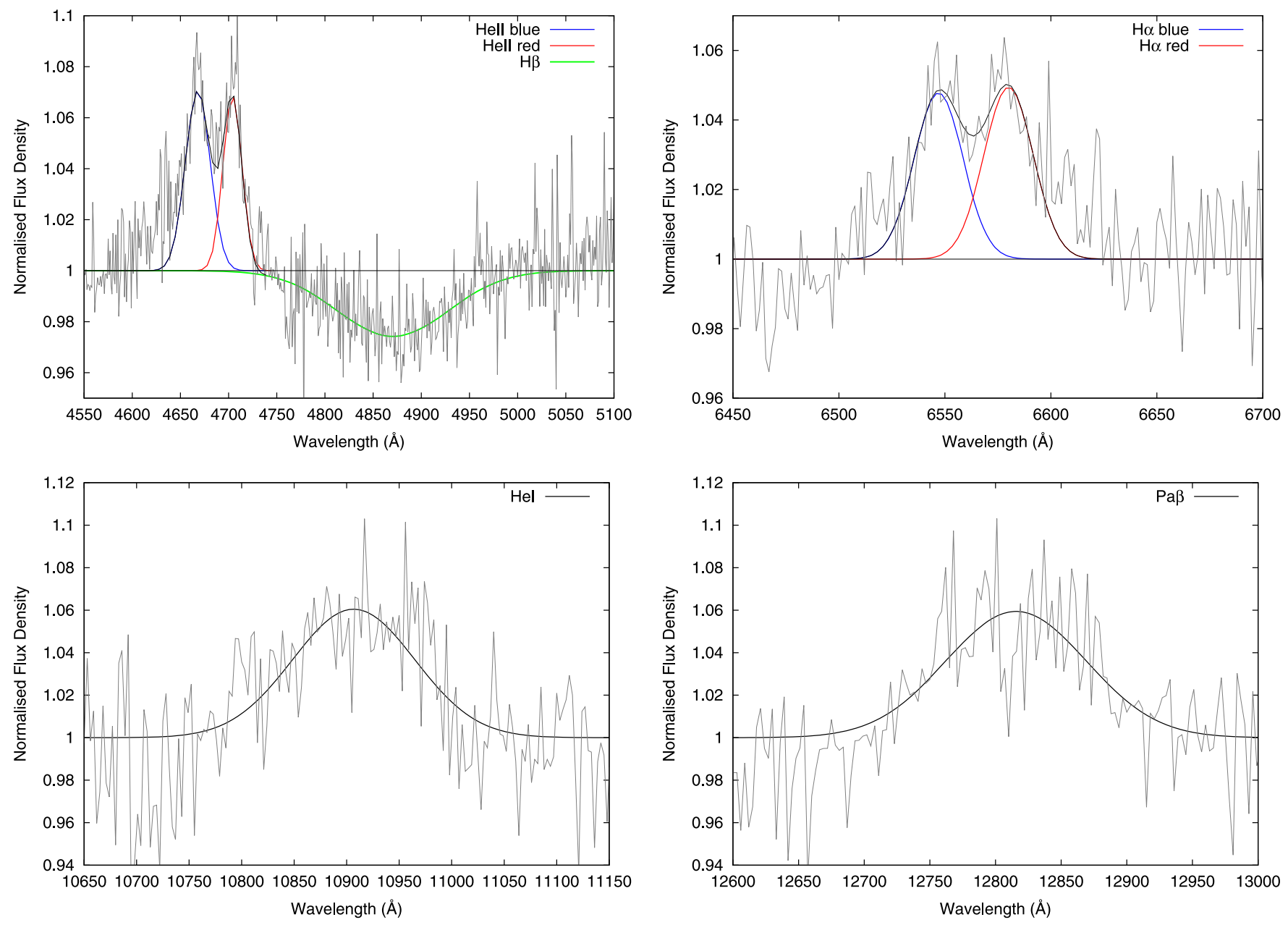

Figure 3. Optical and NIR spectroscopic lines detected in the X-shooter spectrum of Swift J1753.5-0127. Top left: double-peaked He II $\lambda 4686$ emission and broad H $\beta$ absorption; top right: double-peaked $\mathrm{H} \alpha$ emission; bottom left: broad $\mathrm{He}$ I $\lambda 10905$ emission, likely double peaked; bottom right: broad Pa $\beta$ emission, likely double peaked.

Table 4

Optical and NIR Lines in the Swift J1753.5-0127 Spectrum

\begin{tabular}{|c|c|c|c|c|c|c|c|}
\hline \multirow{2}{*}{$\frac{\text { Parameter }}{\lambda_{\mathrm{c}}^{\mathrm{a}}}$} & \multicolumn{2}{|c|}{ He II } & \multirow{2}{*}{$\frac{\mathrm{H} \beta}{4859 \pm 7}$} & \multicolumn{2}{|c|}{$\mathrm{H} \alpha$} & \multirow{2}{*}{$\frac{\mathrm{He}_{\mathrm{I}}}{10906 \pm 9}$} & \multirow{2}{*}{$\begin{array}{c}\mathrm{Pa} \beta \\
12815 \pm 6\end{array}$} \\
\hline & $4668 \pm 2$ & $4704 \pm 4$ & & $6547 \pm 2$ & $6580 \pm 2$ & & \\
\hline$\stackrel{\circ}{W}^{\mathrm{b}}$ & $-2.3 \pm 0.5$ & $-1.7 \pm 0.4$ & $+4.1 \pm 1.4$ & $-1.4 \pm 0.2$ & $-1.5 \pm 0.3$ & $-8.8 \pm 1.5$ & $-7.5 \pm 1.2$ \\
\hline FWHM $^{\mathrm{c}}$ & $1948 \pm 199$ & $1481 \pm 183$ & $8762 \pm 1147$ & $1244 \pm 174$ & $1250 \pm 111$ & $3771 \pm 474$ & $2904 \pm 319$ \\
\hline
\end{tabular}

Notes.

${ }^{a}$ Measured wavelength in $\AA$.

${ }^{\mathrm{b}}$ Equivalent widths in $\AA$.

${ }^{\mathrm{c}}$ FWHM in $\mathrm{km} \mathrm{s}^{-1}$, quadratically corrected for instrumental broadening.

${ }^{\mathrm{d}}$ Intrinsic line flux in units of $10^{-15} \mathrm{erg} \mathrm{cm}^{-2} \mathrm{~s}^{-1}$.

FWHMs of $4250 \mathrm{~km} \mathrm{~s}^{-1}$ and $2450 \mathrm{~km} \mathrm{~s}^{-1}$, peak-to-peak separations of $2690 \mathrm{~km} \mathrm{~s}^{-1}$ and $1650 \mathrm{~km} \mathrm{~s}^{-1}$, and total equivalent widths of $4.3 \AA$ and $3.6 \AA$ for $\mathrm{He}$ II and $\mathrm{H} \alpha$, respectively. In our spectrum, we also report the two emission lines, which are still double peaked, and we fitted their profiles with two Gaussians. Our measurements are in agreement with the values given in Neustroev et al. (2014), and we derive peak-to-peak separations of $2304 \pm 286 \mathrm{~km} \mathrm{~s}^{-1}$ and $1509 \pm 129 \mathrm{~km} \mathrm{~s}^{-1}$ for $\mathrm{He}$ II and $\mathrm{H} \alpha$, respectively. We also report a very broad trough longward of $\mathrm{He}$ II $\left(\mathrm{FWHM} \sim 8800 \mathrm{~km} \mathrm{~s}^{-1}\right)$ that is present in all the individual spectra and that we tentatively associate with $\mathrm{H} \beta$. Such Balmer absorption lines have been detected in other microquasars (e.g., Callanan et al. 1995; Bianchini et al. 1997; Soria et al. 2000; Dubus et al. 2001; Rahoui et al. 2014) and are thought to originate in the viscous accretion disk (La Dous 1989). In the NIR, we report for the first time the presence of very broad emission lines of $\mathrm{He}$ I and $\mathrm{Pa} \beta$ centered at $10906 \AA$ and $12815 \AA$, respectively. We believe that their profiles are double peaked, although double-Gaussian fits are statistically not required for either of them owing to low signal-to-noise ratios $(\mathrm{S} / \mathrm{Ns})$. Their FWHMs, measured through single-Gaussian fitting, are roughly on par with the total FHWMs of the optical emission 
Table 5

Equivalent Widths $\stackrel{\circ}{W}$ - Converted into $E(B-V)$ ISM Reddening Values —of Selected ISM Absorption Lines Present in the Swift J1753.5-0127 Spectrum

\begin{tabular}{lcc}
\hline \hline Line & $\stackrel{\circ}{W}$ & $E(B-V)$ \\
\hline DIB $\lambda 5780$ & $0.27 \pm 0.02$ & $0.47 \pm 0.03^{\mathrm{a}}$ \\
Na I D2 $\lambda 5890$ & $0.71 \pm 0.05$ & $0.42 \pm 0.04^{\mathrm{b}}$ \\
Na I D1 $\lambda 5896$ & $0.57 \pm 0.04$ & $0.47 \pm 0.05^{\mathrm{b}}$ \\
K I $\lambda 7699$ & $0.12 \pm 0.02$ & $0.43 \pm 0.07^{\mathrm{c}}$ \\
\hline
\end{tabular}

Notes. We derive an average value $E(B-V)=0.45 \pm 0.02$.

a Using the $\stackrel{\circ}{W}$ to $E(B-V)$ conversion factor given in Jenniskens \& Desert (1994).

${ }^{\mathrm{b}}$ Using the $\stackrel{\circ}{W}$ to $E(B-V)$ relation given in Poznanski et al. (2012).

${ }^{\text {c }}$ Using the $\stackrel{\circ}{W}$ to $E(B-V)$ relation given in Munari \& Zwitter (1997).

lines, hinting at similar locations in the accretion disk. Besides the features discussed above, no other emission lines are detected.

\subsection{The Interstellar Reddening}

We surveyed the X-shooter spectrum to find interstellar lines relevant for the determination of the reddening suffered by Swift J1753.5-0127 along its line of sight. We unambiguously identified four of them with $\mathrm{S} / \mathrm{N}$ high enough to be used to that purpose: the diffuse interstellar band (DIB) at $5780 \AA$, the D2 and D1 Na I doublet at 5890 and $5896 \AA$, respectively, and the $\mathrm{K}_{\mathrm{I}}$ line at $7699 \AA$. We measured their respective equivalent widths through Gaussian fitting and used various $\dot{W}$ versus $E(B-V)$ relations-Jenniskens \& Desert (1994) for DIB $\lambda 5780$, Munari \& Zwitter (1997) for K I, and Poznanski et al. (2012) for the $\mathrm{NaI}$ doublet-to derive four $E(B-V)$ measurements that we averaged to yield $E(B-V)=0.45 \pm$ 0.02 (see Table 5). This result is similar to previous measurements, in particular, $E(B-V)=0.42 \pm 0.02$ found in Durant et al. (2009) and $E(B-V)=0.45 \pm 0.05$ in Froning et al. (2014). Moreover, of relevance for multiwavelength SED modeling, this interstellar reddening value is consistent with a column density between $N_{\mathrm{H}}=(3.08 \pm 0.19) \times$ $10^{21} \mathrm{~cm}^{-2}$ and $N_{\mathrm{H}}=(4.00 \pm 0.24) \times 10^{21} \mathrm{~cm}^{-2}$, using the average total-to-selective extinction ratio $R_{\mathrm{V}}=3.1$ and the relations $N_{\mathrm{H}}=(2.21 \pm 0.09) \times 10^{21} A_{\mathrm{V}}$ and $N_{\mathrm{H}}=(2.87 \pm$ $0.12) \times 10^{21} A_{\mathrm{V}}$ given in Güver \& Özel (2009) and Foight et al. (2015), respectively.

\subsection{The Continuum}

Swift J1753.5-0127 exhibits a very blue spectrum, and the equivalent widths of the NIR emission lines are significantly larger than those at optical wavelengths despite similar or lower intrinsic fluxes. Considering that these features are likely formed in the outer regions of the accretion disk, this points toward the disk as the main contributor to the continuum.

Furthermore, the optical flux level is similar to those reported in previous studies, which is consistent with the very weak average photometric variability of the source. However, Neustroev et al. (2014) reported a flattening of the continuum between 4000 and $5400 \AA$, which is not present in our spectrum. The authors also argue that the optical continuum was variable on a night-to-night timescale, strongly between 4000 and $5400 \AA$, and marginally beyond. To check whether we could detect any similar variations, we extracted all the individual $210 \mathrm{~s}$ X-shooter spectra and compared them; we
Table 6

Best Parameters Obtained from Fits to the Swift J1753.5-0127 Swift/XRT Spectrum with the Two Models TBABS $\times$ COMPTT and TBABS $\times($ DISKBB + COMPTT $)$

\begin{tabular}{lcc}
\hline \hline Parameter & TBABS $\times$ COMPTT & TBABS $\times($ DISKBB \pm COMPTT $)$ \\
\hline$N_{\mathrm{H}}{ }^{\mathrm{a}}$ & $0.14_{-0.03}^{+0.02}$ & $0.35_{-0.09}^{+0.08}$ \\
$k T_{\text {in }}{ }^{\mathrm{b}}$ & $\cdots$ & $0.13 \pm 0.01$ \\
$R_{\text {in }}{ }^{\mathrm{c}}$ & $\ldots$ & $21.7_{-9.8}^{+14.1}$ \\
$k T_{0}{ }^{\mathrm{d}}$ & $0.12 \pm 0.02$ & tied to $k T_{\text {in }}$ \\
$k T_{\mathrm{e}}{ }^{\mathrm{e}}$ & $60($ fixed) & 60 (fixed) \\
$\tau^{\mathrm{f}}$ & $0.91_{-0.07}^{+0.08}$ & $0.76_{-0.08}^{+0.09}$ \\
$\chi_{\mathrm{r}}{ }^{2}$ (dof) & $0.91(173)$ & $0.86(172)$ \\
\hline
\end{tabular}

Notes. The error bars are given at the $90 \%$ confidence level.

${ }^{a}$ Column density in units of $10^{22} \mathrm{~cm}^{-2}$.

b Accretion disk inner temperature (keV).

${ }^{\mathrm{c}}$ Inner radius in units of $\frac{D_{\mathrm{BH}}}{M_{\mathrm{BH}} \sqrt{\cos i}} R_{\mathrm{g}}$, with $i$ the inclination, $D_{\mathrm{BH}}$ the distance in kpc, $M_{\mathrm{BH}}$ the mass in $M_{\odot}$, and $R_{\mathrm{g}}$ the gravitational radius.

d Input soft photon temperature (keV).

e Electron temperature (keV).

${ }^{\mathrm{f}}$ Electron optical depth.

could not find any significant flickering, neither in flux level nor in shape, beyond the noise level. It is not clear whether this can be interpreted as a nonvariability of the continuum at short timescales, or if $210 \mathrm{~s}$ is sufficient to smooth out the variations, like in the GX 339-4 case (Rahoui et al. 2012).

\section{SED MODELING}

In the following, the X-shooter spectrum and UVOT photometric points were corrected for interstellar reddening using the extinction law given in Fitzpatrick (1999) with $E(B-V)=0.45 \pm 0.02$ (see Section 3.2) and a selective-tototal extinction ratio equal to the Galactic average value $R_{\mathrm{V}}=3.1$.

\subsection{The X-Ray Emission}

We fitted the spectrum using Xspec v. 12.8.2, first with an absorbed spherical Comptonization component, TBABS $\times$ COMPTT (Titarchuk 1994). We used the abundances given in Wilms et al. (2000) and fixed the electron temperature to $60 \mathrm{keV}$ after confirming that this parameter was not constrained (see T15 for a measurement of $k T_{\mathrm{e}}$ from our 2014 April NuSTAR observations). The best-fit parameters are listed in the left column of Table 6 , and the best-fit model is displayed in the left panel of Figure 4. While the result is satisfactory, with a seed photon temperature $k T_{0} \sim 0.12$, an optical depth $\tau \sim 0.91$, and a reduced $\chi^{2}$ value of 0.91 , the measured bestfit column density $N_{\mathrm{H}}=1.4_{-0.3}^{+0.2} \times 10^{21} \mathrm{~cm}^{-2}$ is a factor of two lower than the expected extinction along the line of sight to Swift J1753.5-0127 (see Section 4.2 for a discussion about the interstellar reddening). This likely means that the soft X-ray flux is underestimated, and we thus performed the fit again, adding an accretion disk component, modeled with DISKBB. We tied the seed photon temperature $k T_{0}$ to the disk temperature and left the electron temperature fixed to $60 \mathrm{keV}$. The best-fit parameters are listed in the right column of Table 6 , and the best-fit spectrum is displayed in the right panel of Figure 4. Adding an accretion disk only slightly improves the fit, with a reduced $\chi^{2}$ of 0.86 . However, the measured column density, $N_{\mathrm{H}}=3.5_{-0.9}^{+0.8} \times 10^{21} \mathrm{~cm}^{-2}$, is much more consistent with the 

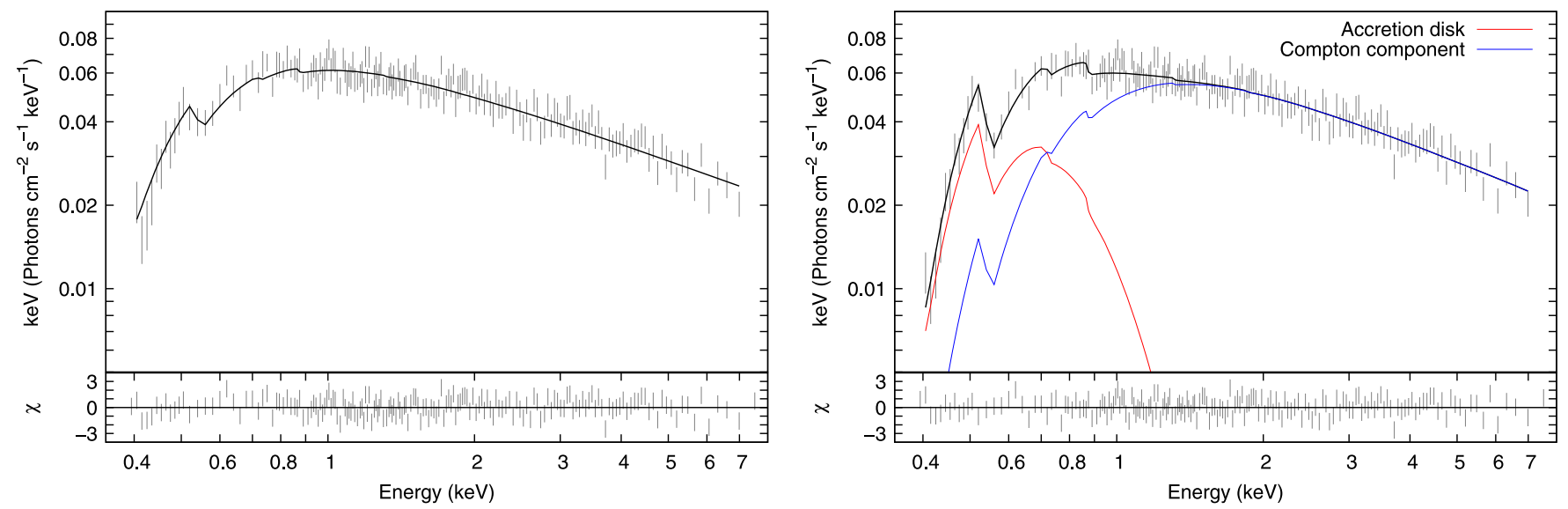

Figure 4. Best fit to the Swift/XRT spectrum of Swift J1753.5-0127 with the models TBABS $\times$ COMPTT (left) and TBABS $\times$ (DISKBB + COMPTT) (right). While the latter model only gives a marginally better result ( $F$-test value of about 10$)$, the inferred column density is more realistic for that celestial position, implying a weak contribution from the accretion disk.
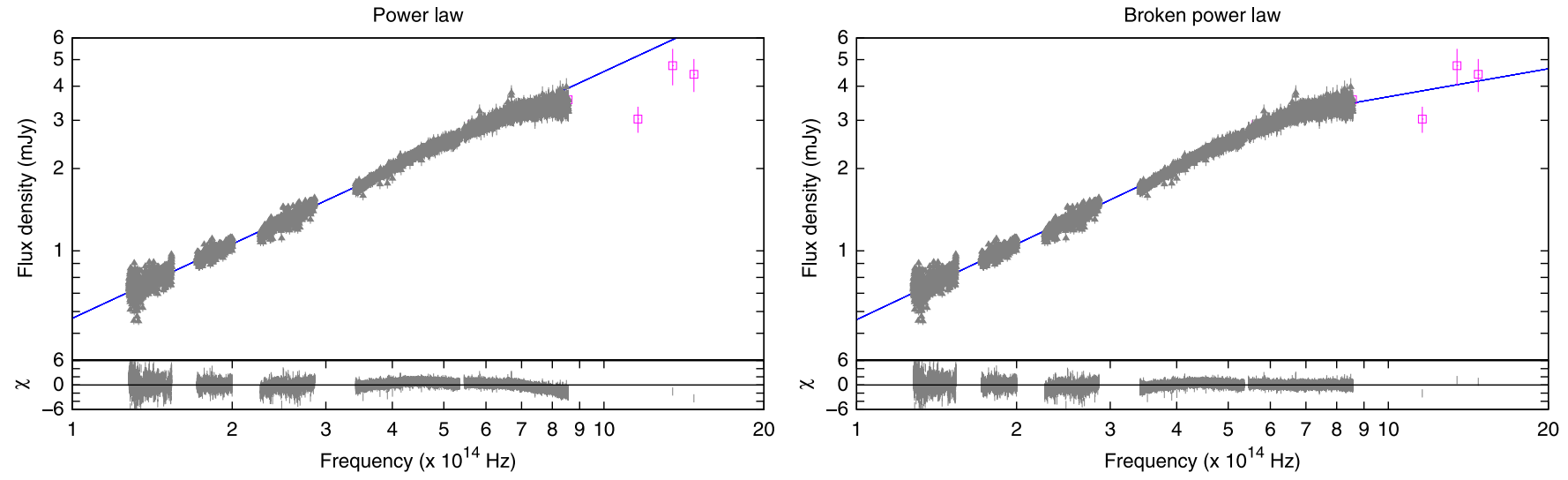

Figure 5. Phenomelogical fit to the UVOT $+X$-shooter data using a power law (left) and a broken power law (right). The best fit is obtained with the broken power law and is consistent with the expected contribution from a viscous accretion disk, although an extra component must be present to account for the flattening in the NIR.

expected interstellar reddening. The seed photon blackbody included in COMPTT is also negligible compared to the disk blackbody emission. We conclude that this implies the presence of an extrasoft component contributing to the X-ray emission of Swift J1753.5-0127, although we stress that our Swift data alone are not sufficient to prove the accretion disk hypothesis.

\subsection{The Optical and NIR Emission}

Froning et al. (2014) showed that the UV, optical, and NIR emission of Swift J1753.5-0127 during their 2012 October 2 observations was likely due to a strongly truncated accretion disk, with the presence of a possible NIR excess. The authors also argued that the simultaneous Swift spectrum was perfectly modeled with an absorbed power law without any need for a disk component. In our case, the presence of double-peaked emission lines in the very blue $\mathrm{X}$-shooter spectrum and the lack of short-timescale variability of the continuum also favor the accretion disk as the main contributor to the optical and NIR emission. This is confirmed by the phenomelogical modeling of the UVOT $+\mathrm{X}$-shooter continuum with a broken power law (displayed in Figure 5; see Table 7 for the best-fit parameters). Beyond the break, the spectral index is roughly consistent with the canonical value, $1 / 3$, expected from a viscous accretion disk (Shakura \& Syunyaev 1973). Below, the spectral index is
Table 7

Best Parameters Obtained from Fits to the Swift J1753.5-0127 X-Shooter Spectrum with a Power Law and a Broken Power Law

\begin{tabular}{lcc}
\hline \hline Parameter & Power Law & Broken Power Law \\
\hline$\alpha_{1}{ }^{\mathrm{a}}$ & $0.902 \pm 0.002$ & $0.918 \pm 0.002$ \\
$\nu_{\mathrm{b}}{ }^{\mathrm{b}}$ & $\ldots$ & $6.74_{-0.11}^{+0.10} \times 10^{14}$ \\
$\alpha_{2}{ }^{\mathrm{c}}$ & $\ldots$ & $0.25 \pm 0.03$ \\
$\chi_{\mathrm{r}}{ }^{2}$ (dof) & $1.17(6170)$ & $0.97(6168)$ \\
\hline
\end{tabular}

Notes. The error bars are given at the $90 \%$ confidence level.

${ }^{a}$ Spectral index of the power law below the break, if any.

${ }^{\mathrm{b}}$ Spectral break location, in $\mathrm{Hz}$.

${ }^{\mathrm{c}}$ Spectral index of the power law beyond the break.

flatter than a typical Raleigh-Jeans tail, pointing toward the contribution of at least one extra component.

In contrast to Froning et al. (2014), our derived $0.3-8 \mathrm{keV}$ flux of Swift J1753.5-0127 is $4.35 \times 10^{-10} \mathrm{erg} \mathrm{cm}^{-2} \mathrm{~s}^{-1}$, i.e., about $80 \%$ larger than their reported value, and we argue that an extrasoft X-ray component is required. It is therefore likely that the disk is responsible for both this soft $\mathrm{X}$-ray excess and a significant fraction of the optical and NIR emission. Nonetheless, as seen in Figure 6, the accretion disk continuum as derived from the X-ray fit only is unable to account for the 


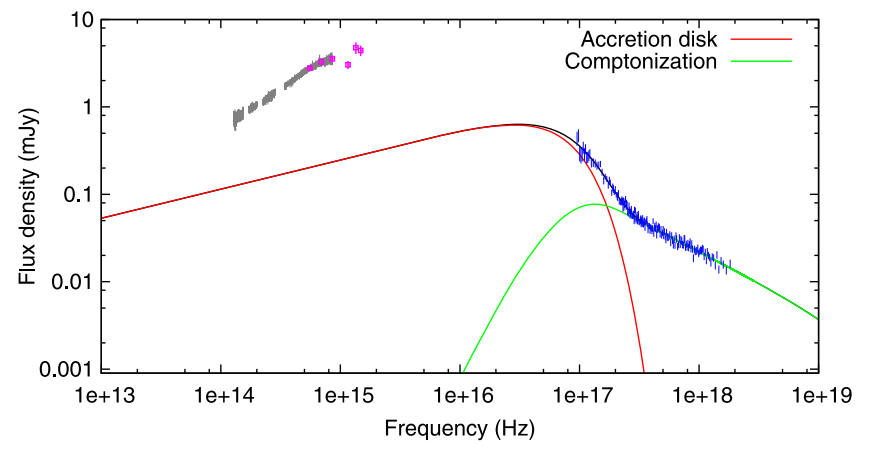

Figure 6. Fit to the Swift/XRT+UVOT and X-shooter data using the X-rayonly model presented in Section 4.1. The disk emission clearly underestimates the low-energy flux level, which justifies the simultaneous fitting of the X-ray to NIR SED to properly derive the accretion disk parameters.

UVOT and X-shooter flux level, and a complete characterization of the accretion disk properties requires a simultaneous modeling of the Swift/XRT+UVOT and X-shooter data.

\subsection{Broadband SED}

We therefore fitted the radio, UV/optical/NIR, and X-ray SED of Swift J1753.5-0127 with several models that include an accretion disk. We chose DISKIR, introduced in Gierliński et al. (2008), for the following reasons: (1) it is designed to selfconsistently fit optical/NIR and X-ray data as it includes a Comptonization component; (2) it takes X-ray irradiation phenomena into account; and (3) it allows a derivation of the outer radius of the accretion disk. We also fixed a few parameters of the DISKIR model after several attempts failed to constrain them. As previously mentioned, the electron temperature of the Comptonization component was fixed to $60 \mathrm{keV}$. The irradiation radius $R_{\text {irr }}$, which represents the size of the inner disk region that is illuminated by the corona, was fixed to to $1.0001 R_{\text {in }}$, the minimum allowed value toward which it was converging. The fraction of hard X-ray emission thermalized in the inner disk, $f_{\text {in }}$, was frozen to the recommended value for the hard state, 0.1 , while the fraction of X-ray emission thermalized in the outer disk, $f_{\text {out }}$, which systematically converged to 0 , was eventually fixed to that value.

\subsubsection{DISKIR ONLY}

We first attempted to fit the X-shooter and XRT-UVOT SED of Swift J1753.5-0127 with DISKIR only. Figure 7 displays the best-fit model, and Table 8 lists the best-fit parameters. It is clear that DISKIR alone is insufficient to describe both the X-ray and optical/NIR data, with a reduced $\chi^{2}$ of 6.1 . Figure 7 also shows the superimposed NIR spectrum of an M4V star at $3 \mathrm{kpc}$, illustrating that no contribution is expected from the companion star, and we can therefore confirm that besides the disk, another nonstellar component must contribute to the X-ray and optical/NIR emission. We also stress that the fit gives a column density $N_{\mathrm{H}}$ consistent with the interstellar reddening and points toward a cold and truncated accretion disk.

\subsubsection{DISKIR and Blackbody}

The phenomenological modeling displayed in Figure 5 and the DISKIR-only fit are consistent with the presence of an excess

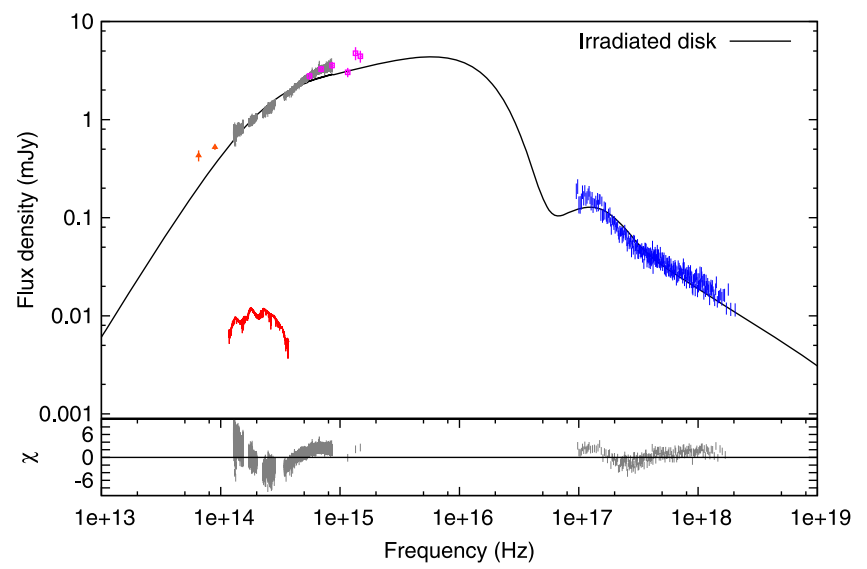

Figure 7. Extinction-corrected VLT/X-shooter+Swift/XRT-UVOT SED of Swift J1753.5-0127 fitted with DISKIR (disk-only case; see Table 8, second column). The WISE flux densities obtained 1 month after our observations (orange) are superimposed but were not part of the fit. We also show the expected contribution of an M4V star at a distance of $3 \mathrm{kpc}$ (magenta).

in the optical and NIR domains. Although it is clear that the emission from the companion star is negligible, the stellar hemisphere facing the $\mathrm{BH}$ could still be irradiated by the X-ray emission, leading to such an excess. Alternatively, a warm dust component similar to those previously detected in other microquasars (see, e.g., Rahoui et al. 2010) could also be present. To test these possibilities, we fit the X-shooter and XRT-UVOT SED of Swift J1753.5-0127 adding a spherical blackbody to DISKIR; the best-fit parameters are listed in the second column of Table 8, and the best-fit SED is displayed in Figure 8 . The addition of the blackbody emission clearly improves the fit, with a reduced $\chi^{2}$ of 0.94 , confirming the presence of an excess in the optical and NIR domains. However, the best-fit parameters are consistent with neither stellar irradiation nor warm dust. Indeed, the best-fit temperature, $2376 \pm 25 \mathrm{~K}$, is too high to be that of warm dust, which sublimes around $1500 \mathrm{~K}$ (see, e.g., Draine 2003), while the best-fit radius, $2.72 \pm 0.03 R_{\odot}$ for a distance of $3 \mathrm{kpc}$, is too large to be that of an $\mathrm{M}$ dwarf star, typically smaller than $0.6 R_{\odot}$ (Boyajian et al. 2012). As such, the optical and NIR excess must therefore stem from another process.

\subsubsection{DISKIR and Broken Power Law}

Our observations occurred when Swift J1753.5-0127 was in the hard state, and the source is regularly detected at radio frequencies. In particular, its radio flux at $15.4 \mathrm{GHz}$ was measured with AMI at about $290 \mu \mathrm{Jy}$ a few days after our observations (M. Kolehmainen 2015, private communication). It is therefore reasonable to expect that a compact jet contributes to its optical and NIR emission through synchrotron radiation, as seen in many other microquasars (see, e.g., Eikenberry et al. 1998; Corbel \& Fender 2002; Chaty et al. 2003; Russell et al. 2006, 2010). To test this hypothesis, we replaced the blackbody by a broken power law to mimic the bolometric emission of the jet as calculated in Blandford \& Konigl (1979), i.e., a combination of optically thick synchrotron $\left(F_{\nu} \propto \nu^{\alpha_{1} \geqslant 0}\right)$ from the radio domain to a spectral break $\nu_{\mathrm{b}}$ where the synchrotron becomes optically thin $\left(F_{\nu} \propto \nu^{\alpha_{2} \leqslant 0}\right)$. Unfortunately, Swift J1753.5-0127 was not detected at radio frequencies during our observations owing to poor conditions, and we can only rely on radio upper limits to constrain the 
Table 8

Best-fit Parameters Derived from Fitting of Swift J1753.5-0127 X-Shooter+Swift/XRT-UVOT SED with TBABS $\times$ DISKIR and TBABS $\times($ DISKIR + BKNPOw)

\begin{tabular}{|c|c|c|c|c|}
\hline \multirow[t]{2}{*}{ Parameter } & \multirow[t]{2}{*}{ TBABS $\times$ DISKIR } & \multirow[t]{2}{*}{ TBABS $\times($ DISKIR + BBODY $)$} & \multicolumn{2}{|c|}{ TBABS $\times($ DISKIR + BKNPOW $)$} \\
\hline & & & Case 1 & Case 2 \\
\hline$N_{\mathrm{H}}{ }^{\mathrm{a}}$ & $0.32 \pm 0.01$ & $0.30 \pm 0.01$ & $0.21 \pm 0.02$ & $0.29 \pm 0.02$ \\
\hline$k T_{\text {disc }}$ b & $0.027_{-0.001}^{+0.002}$ & $0.014 \pm 0.001$ & $0.037 \pm 0.008$ & $0.015_{-0.001}^{+0.002}$ \\
\hline$\Gamma^{\mathrm{c}}$ & $1.78 \pm 0.02$ & $1.70 \pm 0.03$ & $2.71_{-0.26}^{+0.30}$ & $1.70 \pm 0.03$ \\
\hline$k T_{\mathrm{e}}^{\mathrm{d}}$ & 60 (fixed) & 60 (fixed) & 60 (fixed) & 60 (fixed) \\
\hline$L_{\mathrm{c}} / L_{\mathrm{d}}^{\mathrm{e}}$ & $1.42_{-0.17}^{+0.14}$ & $3.79_{-0.44}^{+0.62}$ & $0.11_{-0.02}^{+0.07}$ & $3.22_{-0.67}^{+0.64}$ \\
\hline$f_{\text {in }}{ }^{\mathrm{f}}$ & 0.10 (fixed) & 0.10 (fixed) & 0.10 (fixed) & 0.10 (fixed) \\
\hline$f_{\text {out }}{ }^{g}$ & 0 (fixed) & 0 (fixed) & 0 (fixed) & 0 (fixed) \\
\hline$R_{\text {in }}{ }^{\mathrm{h}}$ & $(6.03 \pm 0.46) \times 10^{2}$ & $1.71_{-0.15}^{+0.28} \times 10^{3}$ & $4.47_{-0.88}^{+2.88} \times 10^{2}$ & $1.60_{-0.19}^{+0.26} \times 10^{3}$ \\
\hline$R_{\text {out }}^{\mathrm{i}}$ & $345.1_{-23.7}^{+30.3}$ & $63.8_{-8.7}^{+6.7}$ & $223.8_{-66.6}^{+63.9}$ & $57.3_{-7.6}^{+8.3}$ \\
\hline$R_{\mathrm{irr}}^{\mathrm{j}}$ & 1.0001 (fixed) & 1.0001 (fixed) & 1.0001 (fixed) & 1.0001 (fixed) \\
\hline$\alpha_{1}^{\mathrm{k}}$ & $\ldots$ & $\ldots$ & 0.59 (fixed) & $0.10 \pm 0.01$ \\
\hline$\nu_{\mathrm{b}}^{1}$ & $\cdots$ & $\cdots$ & $6.41_{-0.32}^{+0.35} \times 10^{11}$ & $1.88_{-0.04}^{+0.02} \times 10^{14}$ \\
\hline$\alpha_{2}{ }^{\mathrm{m}}$ & $\cdots$ & $\cdots$ & $-0.34 \pm 0.01$ & $-0.60 \pm 0.06$ \\
\hline$T_{\mathrm{BB}}{ }^{\mathrm{n}}$ & $\cdots$ & $2376 \pm 25$ & $\ldots$ & $\ldots$ \\
\hline$R_{\mathrm{BB}}{ }^{\circ}$ & $\cdots$ & $0.91 \pm 0.01$ & $\cdots$ & $\cdots$ \\
\hline$\chi_{\mathrm{r}}^{2}(\mathrm{dof})$ & $6.09(6344)$ & $0.93(6342)$ & $1.11(6344)$ & $0.94(6343)$ \\
\hline
\end{tabular}

Notes. Error bars are given at the $90 \%$ confidence level.

${ }^{a}$ Column density in units of $10^{22} \mathrm{~cm}^{-2}$.

${ }^{\mathrm{b}}$ Temperature of the unilluminated accretion disk in $\mathrm{keV}$.

${ }^{\mathrm{c}}$ Asymptotic power law photon index.

${ }^{\mathrm{d}}$ Electron temperature in $\mathrm{keV}$.

e Ratio of luminosity of the Compton component with respect to the unilluminated disk.

${ }^{\mathrm{f}}$ Fraction of luminosity in the Compton tail that is thermalized in the inner disk.

${ }^{\mathrm{g}}$ Fraction of the total luminosity thermalized in the outer disk.

${ }^{\mathrm{h}}$ Inner radius in units of $\frac{D_{\mathrm{BH}}}{M_{\mathrm{BH}} \sqrt{\cos i}} R_{\mathrm{g}}$, with $i$ the inclination, $D_{\mathrm{BH}}$ the distance in kpc, $M_{\mathrm{BH}}$ the mass in $M_{\odot}$, and $R_{\mathrm{g}}$ the gravitational radius.

${ }^{\mathrm{i}}$ Outer radius in units of $R_{\text {in }}$.

${ }^{\mathrm{j}}$ Radius of the Compton-illuminated disk in units of $R_{\text {in }}$.

${ }^{\mathrm{k}}$ Spectral index of the optically thick synchrotron emission of the compact jet.

${ }^{1}$ Spectral break of the compact jet in Hz.

${ }^{\mathrm{m}}$ Spectral index of the optically thin synchrotron emission of the compact jet.

${ }^{\mathrm{n}}$ Blackbody excess temperature in $\mathrm{K}$.

${ }^{\circ}$ Blackbody excess radius in $R_{\odot} \mathrm{kpc}^{-1}$.

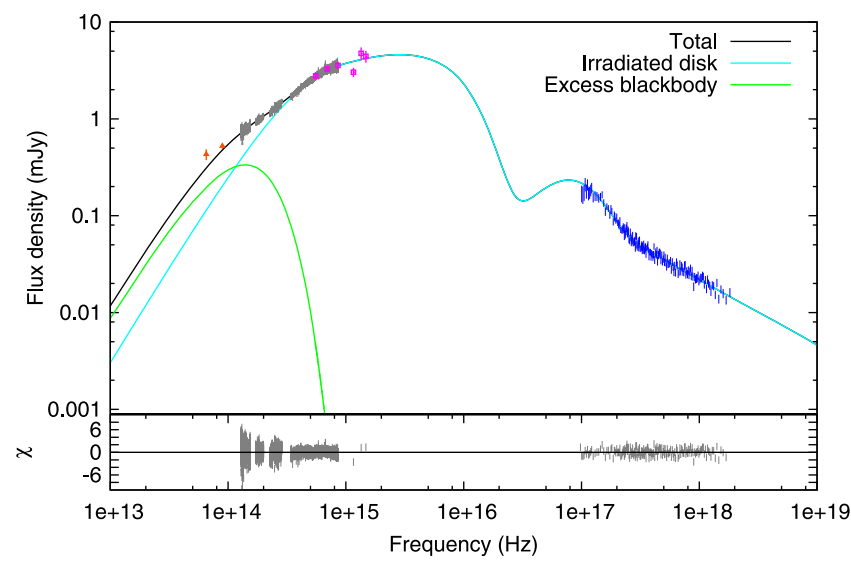

Figure 8. Extinction-corrected VLT/X-shooter + Swift/XRT-UVOT SED of Swift J1753.5-0127 fitted with DISKIR+BBODYRAD (disk plus blackbody case; see Table 8 , second column). The WISE flux densities obtained 1 month after our observations (orange) are superimposed but were not part of the fit.

optically thick spectral index $\alpha_{1}$. Here we therefore consider the following two cases: (1) we fix $\alpha_{1}=0.59$, i.e., the value derived from the radio upper limits at 5.5 and $9 \mathrm{GHz}$; and (2) $\alpha_{1}$ is left free to vary, but the broken power law is forced to match at least the upper limit at $5.5 \mathrm{GHz}$; the derived break

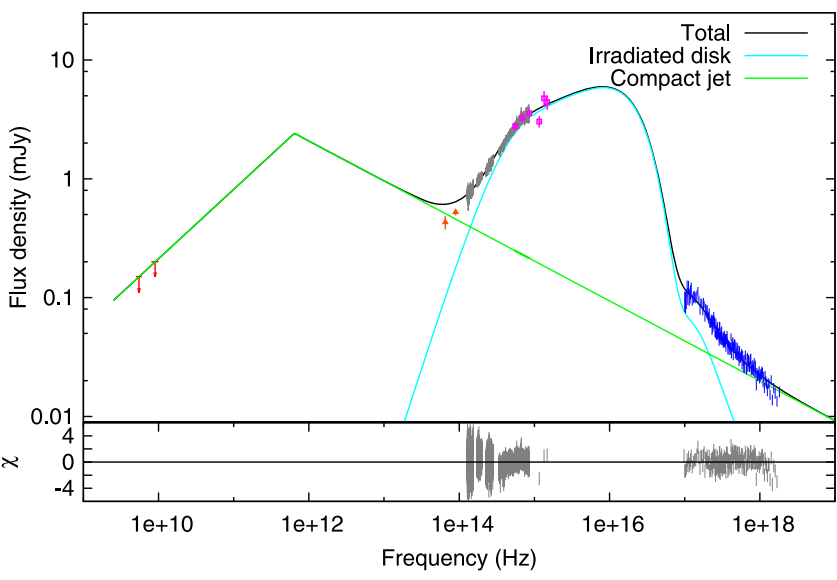

Figure 9. Extinction-corrected ATCA+VLT/X-shooter+Swift/XRT-UVOT SED of Swift J1753.5-0127 fitted with DISKIR+BKNPOw (case 1; see Table 8). Here we fixed the optically thick compact jet spectral index to 0.59 , i.e., the value derived for the two ATCA upper limits at 5.5 and $9 \mathrm{GHz}$. The WISE flux densities obtained 1 month after our observations (orange) are superimposed but were not part of the fit.

frequencies must consequently be considered as lower limits. The best-fit models for the two cases are displayed in Figures 9 and 10, respectively, and Table 8 lists the best-fit parameters. 


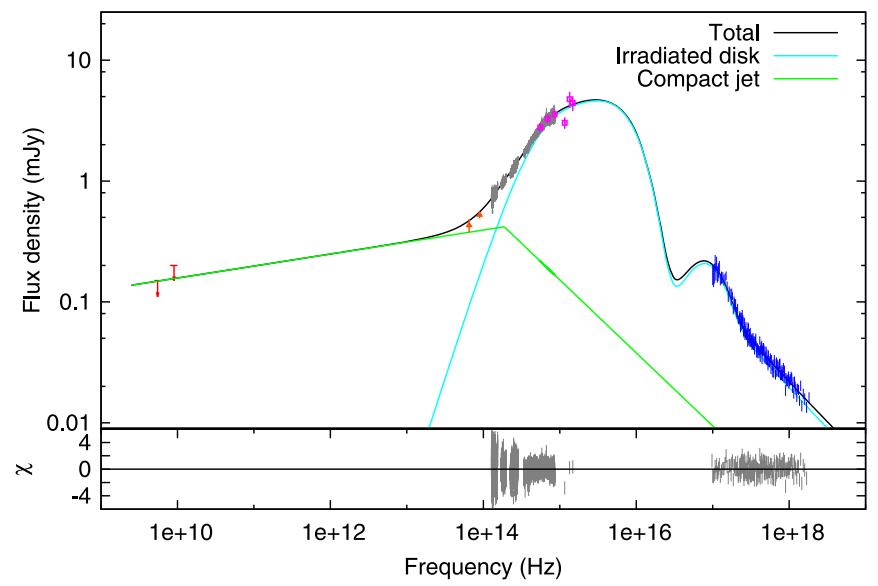

Figure 10. Extinction-corrected ATCA +VLT/X-shooter + Swift/XRT-UVOT SED of Swift J1753.5-0127 fitted with DISKIR+BKNPOw (case 2; see Table 8). Here the optically thick compact jet spectral index is not fixed and the fit lets it be flat. The WISE flux densities obtained 1 month after our observations (orange) are superimposed but were not part of the fit.

Case 2 clearly is the best fit, with a reduced $\chi^{2}$ of 0.94 , and is also the most phenomenologically relevant. Indeed, the derived parameters point toward a weak contribution to the XRT spectrum from a cold and truncated disk, while the coronal emission strongly dominates the X-ray emission. Likewise, the optical and NIR spectrum is well described by the thermal emission from the viscous accretion disk, with an excess due to the synchrotron emission from the jet, both optically thick and thin, as the spectral break is located around $1.88 \times 10^{14} \mathrm{~Hz}$ or $1.60 \mu \mathrm{m}$.We nonetheless stress here that the location of the spectral break in the NIR domain may be an artificial effect of the fitting process due to the lack of information between the radio and $\mathrm{X}$-shooter data. We refer the reader to the next section for a discussion on the reliability of this location and the good agreement between the fit and the WISE flux densities obtained about 1 month after our observations.

In contrast to case 2 , case 1 is worse, with a reduced $\chi^{2}$ of 1.11. More importantly, it is also less relevant from a physical point of view as the derived ratio between the Comptonization component and the unilluminated disk is inconsistent with hard-state spectra of microquasars, with $L_{\mathrm{c}} / L_{\mathrm{d}} \approx 0.11$, whereas a value larger than 1 is expected. A possible explanation is that the fitting process tends to overestimate the contribution from the jet at the expense of that from the corona. Furthermore, while theoretically not ruled out (Blandford \& Konigl 1979), an optically thick synchrotron spectral index of 0.6 has, to our knowledge, never been observed for Swift J1753.5-0127. In Soleri \& Fender (2011), the authors report on several radio spectral indices observed between 2005 and 2009 and find a maximum index of about 0.3 , which is similar to the value we report in T15.

\section{DISCUSSION}

The following discussion is based on our best-fit results for case 2 , and, unless stated otherwise, we assume the following system parameters: (1) $40^{\circ}$ inclination; (2) a $q=0.04$ mass ratio (lower limits; Neustroev et al. 2014); (3) $M_{\mathrm{BH}}=5 M_{\odot}$ $\mathrm{BH}$ mass; (4) $a=1.53 R_{\odot}$ semimajor axis (upper limits; Neustroev et al. 2014); and (5) $3 \mathrm{kpc}$ distance, which roughly corresponds to the expected value for a $5 M_{\odot} \mathrm{BH}$ (Froning et al. 2014). Using the approximate formulae given in
Eggleton (1983) and Frank et al. (2002), we derive a Roche lobe radius $R_{\mathrm{L}} \sim 0.64 a=6.81 \times 10^{10} \mathrm{~cm}$, a tidal radius $R_{\text {tide }} \sim 0.58 a=6.18 \times 10^{10} \mathrm{~cm}$, and a circularization radius $R_{\text {circ }} \sim 0.46 a=4.90 \times 10^{10} \mathrm{~cm}$. Finally, in the Keplerian approximation, the velocity of the accretion disk region that most contributes to a given Gaussian line is related to its FWHM in $\mathrm{km} \mathrm{s}^{-1}$ as

$$
V=\frac{\text { FWHM }}{2 \times \sqrt{\ln 2} \times \sin i} \mathrm{~km} \mathrm{~s}^{-1} .
$$

If the line is double peaked, we also can derive the Keplerian velocity from the peak-to-peak separation $\Delta v$ following

$$
V=\frac{\Delta v}{2 \times \sin i} \mathrm{~km} \mathrm{~s}^{-1}
$$

The Keplerian radius of the region that most contributes to the lines is then given by

$$
R=\frac{c^{2}}{V^{2}} R_{\mathrm{g}},
$$

where $R_{\mathrm{g}}=G M_{\mathrm{BH}} / c^{2}$ is the gravitational radius.

\subsection{The Accretion Disk Properties}

All our SED fits listed in Figure 10 point toward a very truncated accretion disk. From our best fit, we infer an inner radius $R_{\text {in }} \sim 1097 R_{\mathrm{g}}=8.2 \times 10^{8} \mathrm{~cm}$ and an outer radius $R_{\text {out }} \sim 6.3 \times 10^{4} R_{\mathrm{g}}=4.66 \times 10^{10} \mathrm{~cm}$ for case 2 . If we assume that the trough detected in the optical spectrum is $\mathrm{H} \beta$ absorption from the disk, then Equations (1) and (3) also lead to $R_{\mathrm{in}} \leqslant 1343 R_{\mathrm{g}}$. Likewise, assuming that $\mathrm{H} \alpha$ originates from the outer accretion disk, we infer $R_{\text {out }} \sim 6.6 \times$ $10^{4} R_{\mathrm{g}}=4.89 \times 10^{10} \mathrm{~cm}$ from both its FWHM and peak-topeak separation. Both our SED modeling and X-shooter spectrum are therefore consistent with each other and point toward a small and very truncated accretion disk, with $R_{\text {in }} \sim 10^{3} R_{\mathrm{g}}$. Such a large truncation is possible for an ADAF-like flow, but it is thought to be typical of the quiescent state of microquasars, during which their luminosity drops below $10^{-5} L_{\text {edd }}$ (Narayan \& McClintock 2008). In contrast, the Swift J1753.5-0127 bolometric luminosity during our observation is about $0.005 L_{\text {edd }}$, more typical of a relatively faint hard state. Interestingly, another outlier, GRO J0422+32, was found to have an even larger disk/ADAF transition radius (Esin et al. 1998); this might hint at the presence of very large truncations in the hard state of outliers.

The low number of emission lines, likely all double peaked, in the spectrum of Swift J1753.5-0127 is in stark contrast to the wealth of emission lines, mostly single peaked, detected in the hard-state optical/NIR spectra of other microquasars (see, e.g., Bandyopadhyay et al. 1997; Wu et al. 2001; Rahoui et al. 2014). Outer regions of accretion disks can be directly illuminated by the hard X-ray emission from the Comptonization component and/or the compact jets in the hard state, resulting in strong UV/optical/NIR excess. The paucity of emission lines and the fact that a significant fraction of the $\mathrm{X}$-shooter continuum can be explained solely by the thermal emission from the viscous accretion disk therefore hint at a very low level of outer accretion disk irradiation.

Possible reasons are the low X-ray luminosity or the outer accretion disk not being flared up. However, even a weak X-ray illumination of the accretion disk chromosphere is thought to 
create an inflated envelope (Begelman et al. 1983) that, in the hard state, would likely play an important role in reflecting a significant fraction of the X-ray emission back to the outer regions, where it would be thermalized (Begelman \& McKee 1983; Jimenez-Garate et al. 2002; Gierliński et al. 2009). Following Begelman et al. (1983), such an envelope could exist in a quasi-hydrostatic equilibrium at any radius $R$ in the disk if $T_{\mathrm{c}}<T_{\mathrm{g}}$, where

$$
T_{\mathrm{c}}=\frac{1}{4 k_{\mathrm{B}} L_{\mathrm{X}}} \int_{\nu_{1}}^{\nu_{\mathrm{n}}} h \nu L_{\nu} d \nu \mathrm{K}
$$

is the Compton temperature, with $L_{\mathrm{X}}=\int_{\nu_{1}}^{\nu_{\mathrm{n}}} L_{\nu} d \nu$ the irradiating X-ray luminosity, and

$$
T_{\mathrm{g}}=\frac{2}{3} \frac{G M_{\mathrm{BH}} m_{\mathrm{p}}}{k_{\mathrm{B}} R} \mathrm{~K}
$$

is the escape temperature. Equating Equations (4) and (5) leads to the typical radius $R_{\mathrm{c}}$ within which the envelope is in quasihydrostatic equilibrium,

$$
R_{\mathrm{c}}=\frac{2}{3} \frac{m_{\mathrm{p}} c^{2}}{k_{\mathrm{B}} T_{\mathrm{c}}} R_{\mathrm{g}}
$$

also called the Compton radius. For the Swift $\mathrm{J} 1753.5-0127$ parameters and $\mathrm{X}$-ray emission, this results in $T_{\mathrm{c}} \approx 2.8 \times 10^{8} \mathrm{~K}$ and $R_{\mathrm{c}} \approx 2.6 \times 10^{4} R_{\mathrm{g}}$. In the GX 339-4 case, in which a high level of X-ray irradiation is found (Rahoui et al. 2014), the same calculations lead to $T_{\mathrm{c}} \approx 6.6 \times 10^{7} \mathrm{~K}$ and $R_{\mathrm{c}} \approx 1.2 \times 10^{5} \quad R_{\mathrm{g}}$ compared to $R_{\text {out }} \approx 1.8 \times 10^{5} R_{\mathrm{g}}$. The quasi-hydrostatic envelope therefore covers about $40 \%$ of the accretion disk in Swift J1753.5-0127 versus 67\% in GX 339-4. Even if other parameters may account for the low level of thermalized hard X-ray photons, a reasonable explanation is thus that a significant fraction of hard X-ray photons do not reach the outer regions of the accretion disk. It is also interesting that the Compton temperature is more than four times larger in Swift J1753.5-0127 than GX 339-4 despite the latter being 10 times more X-ray luminous than the former $\left(0.005 L_{\text {edd }}\right.$ versus $0.05 L_{\text {edd }}$, respectively). In the X-ray spectrum of GX 339-4 in the hard state, a significant contribution from the accretion disk is present (Rahoui et al. 2012). In contrast, the Swift J1753.5-0127 X-ray emission mostly stems from Comptonization (see the next section and T15). This actually illustrates the fact that Compton heating is as sensitive to the hardness of the $\mathrm{X}$-ray spectrum as to the X-ray luminosity itself (see Equation (4)). This is why bright hard states of microquasars could be more favorable than soft states to Compton heating of accretion disk chromospheres and the presence of thermally driven winds launched from the envelope (see Rahoui et al. 2014 for a discussion on GX 339-4). Nonetheless, a necessary condition is that the irradiating X-ray emission is at least twice as bright as a critical luminosity defined as

$$
L_{\mathrm{cr}}=\frac{288}{\sqrt{T_{\mathrm{c}}}} L_{\mathrm{edd}} .
$$

The presence of Compton-heated winds can therefore be ruled out for Swift J1753.5-0127 as $L_{\mathrm{cr}} \approx 0.02 L_{\mathrm{edd}}$, to compare to a luminosity of about $0.005 L_{\text {edd }}$.

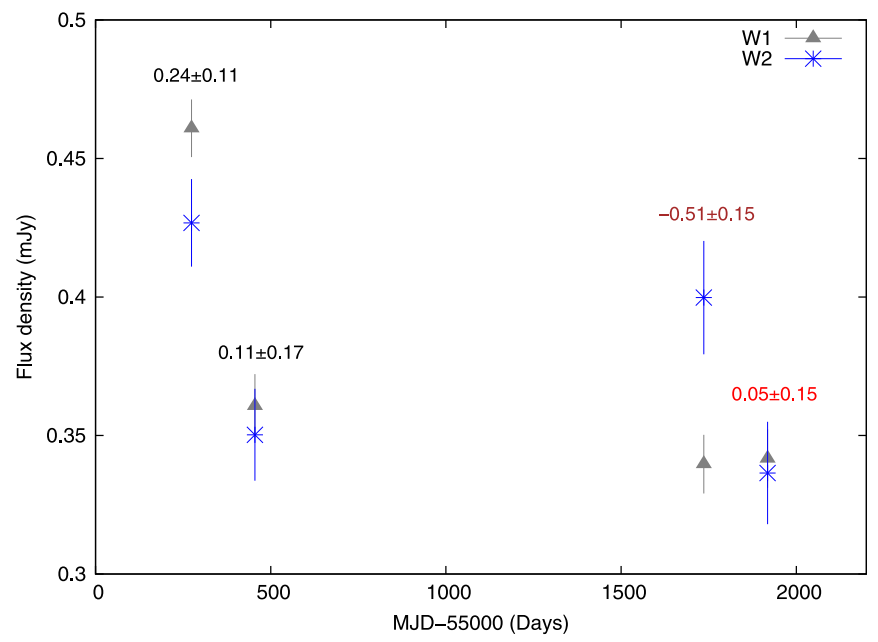

Figure 11. Dereddened WISE W1 and W2 flux densities obtained on MJD 55,274, MJD 55,454, MJD 56,736, and MJD 56,918. They are corrected for the contribution from the accretion disk as measured in case 2 to trace the compact jet emission. We also give the spectral index derived at each epoch, including 20 days before our April observations (brown; see T15) and 1 month after the August observations reported in this paper (red).

\subsection{The Compact Jet Emission and the Origin of the X-Ray Emission}

The SED modeling points toward the detection of the compact jet in the NIR via, at least, an optically thin synchrotron component well constrained by the presence of an NIR excess. But whether optically thick synchrotron emission contributes to the optical/NIR domain depends on the frequency turnover $\nu_{\mathrm{b}}$ and the nondetection of the source in the radio domain strongly limits our capacity to constrain its location, although it is very likely beyond $6.4 \times 10^{11} \mathrm{~Hz}$. We note that the W1 and W2 WISE flux densities obtained about 1 month after our observations are almost consistent with our case 2 scenario, for which the spectral break is located in the NIR domain, beyond $1.9 \times 10^{14} \mathrm{~Hz}$. If a coincidence cannot be ruled out, this could also mean that the average compact jet emission was relatively constant over a few months and that case 2 properly describes the jet properties. To check to which extent such a weakly inverted infrared emission is common, we measured the mid-infrared slopes at other epochs based on the archival WISE data. Figure 11 displays the dereddened W1 and W2 WISE flux densities of Swift J1753.5-0127 for MJD 55,274, MJD 55,454, MJD 56,736, and MJD 56,918; note that we do not include the measurements for MJD 55,456 as they are similar to those on MJD 55,454. These flux densities were first corrected for the accretion disk contribution as measured in our fits. We thus assume that the accretion disk emission is relatively constant, which is reasonable considering the low variability of the optical magnitudes of the source since the decay of the initial outburst (see Figure 1 in Shaw et al. 2013). Assuming that no other component, in particular dust, contributed (see, e.g., Rahoui et al. 2010; Chaty \& Rahoui 2012), these flux densities therefore trace the compact jet emission, and we can estimate the spectral index of its synchrotron radiation at each epoch. Besides MJD 56,918 data that we already show in our fits, we find that this index was also positive and consistent with optically thick synchrotron on MJD 55,274 and MJD 55,454 (hence MJD 55,456), which strengthens the possibility that the spectral break was indeed located in the NIR domain during our observations. In contrast, 
the spectral index is negative and consistent with optically thin synchrotron only once, on MJD 56,736. Interestingly, these WISE data were obtained about 20 days before our 2014 April observations. This is consistent with the results presented in T15, where we find that the compact jet synchrotron emission in the WISE bands was optically thin, and where we report a spectral break in the range $2.4 \times 10^{10}-3.6 \times 10^{12} \mathrm{~Hz}$, derived with much better radio constraints when the X-ray luminosity of Swift J1753.5-0127 was about 50\% lower. It is therefore very likely that the turnover shifted to higher frequencies in August compared to April, especially considering that the NIR excess was much larger, hinting at a more important contribution from the compact jet. Such behavior may be expected as $\nu_{\mathrm{b}} \propto L_{\mathrm{X}}^{1 / 3}$ or $\nu_{\mathrm{b}} \propto L_{\mathrm{X}}^{2 / 3}$ whether we consider a radiatively efficient or inefficient flow, respectively (Falcke \& Biermann 1995; Heinz \& Sunyaev 2003). However, Russell et al. (2013) did not find any correlation between the spectral break frequency and the X-ray luminosity, and other parameters, such as the base radius of the jet and/or the strength of the magnetic field, must be considered (see, e.g., Chaty et al. 2011; Gandhi et al. 2011); a detailed analysis is beyond the scope of this paper, and we refer to T15 for a comprehensive discussion of the compact jet energetics.

Nonetheless, whichever spectral break frequency we consider, it is clear that the optically thin synchrotron from the compact jet marginally contributes to the soft X-ray spectrum, which almost completely stems from the Comptonization component. This is a property that Swift J1753.5-0127 shares with other outliers, such as XTE J1650-500 (Corbel et al. 2004), XTE J1720-318, (Chaty \& Bessolaz 2006), and Cygnus X-1 (Rahoui et al. 2011). Outliers are characterized by a steeper X-ray/radio correlation $L_{\mathrm{R}} \propto L_{\mathrm{X}}^{\xi}$ so that the radio flux density for a given $\mathrm{X}$-ray luminosity is systematically lower than what is expected. A possible explanation, dubbed the radio-quiet hypothesis, is that compact jets in outliers are intrinsically weaker. On the other hand, Coriat et al. (2011) estimated $\xi \approx 1.4$ for H1743-322 and proposed that the accretion flow in outliers may rather be radiatively efficient $\left(L_{\mathrm{X}} \propto \dot{M}\right)$, as opposed to "standard" microquasars, for which it is radiatively inefficient $\left(L_{\mathrm{X}} \propto \dot{M}^{2-3}\right)$; this is the X-ray-bright hypothesis. An alternative explanation still consistent with the $\mathrm{X}$-ray-bright phenomenon is, however, the presence of an extra component in the soft X-ray band that is not related to the jet but contributes to the X-ray emission, effectively creating an $\mathrm{X}$-ray excess. What this component could be is a matter of debate, but the geometry of the system, i.e., a highly truncated accretion disk and a large ADAF-like Comptonization component, could be consistent with the presence of the condensation-induced residual inner accretion disk predicted by evaporation models (Liu \& Meyer-Hofmeister 2001; Liu et al. 2007; Meyer-Hofmeister \& Meyer 2014). These residual disks could be irradiated by the hard X-rays but would only carry a very small fraction of the total accreted material. They could in consequence be responsible for an excess soft X-ray emission while contributing very little to the fueling of the compact jet. Finally, Coriat et al. (2011) showed that H1743-322 transitions back to the "standard" radio/ $\mathrm{X}$-ray track below a critical luminosity, and this behavior was also observed in XTE J1550-564 (Russell et al. 2010) and XTE J1752-533 (Ratti et al. 2012). This could also be explained by the presence of a residual inner disk, as the model predicts that such a disk can only exist in the range of about
(0.001-0.02) $L_{\text {Edd }}$ (see Meyer-Hofmeister \& Meyer 2014 for a more detailed discussion on the effects of residual disks on the $\mathrm{X}$-ray/radio correlation in microquasars).

\section{SUMMARY AND CONCLUSION}

We have presented a multiwavelength study of the outlier Swift J1753.5-0127 that focused primarily on its X-shooter spectrum. Based on the spectral analysis of double-peaked emission lines and SED modeling, we find that the optical and NIR emission of the source mostly stems from the thermal radiation of a very truncated accretion disk, with $R_{\text {in }} \sim 1000 R_{\mathrm{g}}$. There also is a significant contribution from the compact jet, the spectral break of which may be located in the NIR. Nonetheless, its optically thin synchrotron radiation cannot account for the soft X-ray emission of Swift J1753.5-0127, which mainly originates from a very large ADAF-like Comptonization component. Finally, the level of irradiation of the outer accretion disk is low, and we propose that this may be due to the relative compactness of a hard X-ray-induced envelope above the disk plane that cannot reflect enough X-ray photons back to the outer regions.

Although the presence of strongly truncated accretion disks in the hard state of microquasars is still a matter of debate, our results and those presented in T15 are consistent with previous multiwavelength studies of Swift J1753.5-0127 that all hint at large inner radii (see, e.g., Zhang et al. 2010; Froning et al. 2014). On the other hand, several authors claimed the detection of a cold accretion disk extending to the ISCO (Reis et al. 2009; Reynolds et al. 2010; Mostafa et al. 2013), based solely on X-ray data, in particular the detection of iron emission lines. These seemingly contradictory results can be reconciled if we consider the presence of both a strongly truncated disk and a residual one created by condensation of the Comptonization component. This illustrates the importance of quasi-simultaneous X-ray and optical/NIR data sets to constrain the properties of accretion disks. We therefore recommend further multiwavelength observations of outliers to understand to which extent the presence of residual inner disks may be a common pattern.

We thank the referee for very insightful and constructive comments. F.R. thanks the ESO staff who performed the service observations. J.A.T. acknowledges partial support from NASA under Swift Guest Observer grants NNX13AJ81G and NNX14AC56G. S.C. acknowledges the financial support from the UnivEarthS Labex program of Sorbonne Paris Cité (ANR10-LABX-0023 and ANR-11-IDEX-0005-02) and from the CHAOS project ANR-12-BS05-0009 supported by the French Research National Agency. E.K. acknowledges support from the TUBITAK BIDEB 2219 program. This work was supported by the Spanish Ministerio de Economía y Competitividad (MINECO) under grant AYA2013-47447-C3-1-P (S. M.). This research has made use of data obtained from the High Energy Astrophysics Science Archive Research Center (HEASARC), provided by NASA's Goddard Space Flight Center. This publication also makes use of data products from NEOWISE, which is a project of the Jet Propulsion Laboratory/California Institute of Technology, funded by the Planetary Science Division of the National Aeronautics and Space Administration. The Australia Telescope Compact Array is part of the Australia Telescope, which is funded by the Commonwealth of Australia for operation as a National 
Facility managed by CSIRO. This research has made use of NASA's Astrophysics Data System and of the SIMBAD and VizieR databases operated at CDS, Strasbourg, France.

\section{REFERENCES}

Bandyopadhyay, R., Shahbaz, T., Charles, P. A., van Kerkwijk, M. H., \& Naylor, T. 1997, MNRAS, 285, 718

Begelman, M. C., \& McKee, C. F. 1983, ApJ, 271, 89

Begelman, M. C., McKee, C. F., \& Shields, G. A. 1983, ApJ, 271, 70

Bianchini, A., della Valle, M., Masetti, N., \& Margoni, R. 1997, A\&A, 321, 477

Blandford, R. D., \& Konigl, A. 1979, ApJ, 232, 34

Boyajian, T. S., von Braun, K., van Belle, G., et al. 2012, ApJ, 757, 112

Burrows, D. N., Hill, J. E., Nousek, J. A., et al. 2005, SSRv, 120, 165

Cadolle Bel, M., Ribó, M., Rodriguez, J., et al. 2007, ApJ, 659, 549

Callanan, P. J., Garcia, M. R., McClintock, J. E., et al. 1995, ApJ, 441, 786

Chaty, S., \& Bessolaz, N. 2006, A\&A, 455, 639

Chaty, S., Dubus, G., \& Raichoor, A. 2011, A\&A, 529, A3

Chaty, S., Haswell, C. A., Malzac, J., et al. 2003, MNRAS, 346, 689

Chaty, S., \& Rahoui, F. 2012, ApJ, 751, 150

Corbel, S., Coriat, M., Brocksopp, C., et al. 2013, MNRAS, 428, 2500

Corbel, S., \& Fender, R. P. 2002, ApJL, 573, L35

Corbel, S., Fender, R. P., Tomsick, J. A., Tzioumis, A. K., \& Tingay, S. 2004, ApJ, 617, 1272

Corbel, S., Fender, R. P., Tzioumis, A. K., et al. 2000, A\&A, 359, 251

Coriat, M., Corbel, S., Prat, L., et al. 2011, MNRAS, 414, 677

Draine, B. T. 2003, ARA\&A, 41, 241

Dubus, G., Kim, R. S. J., Menou, K., Szkody, P., \& Bowen, D. V. 2001, ApJ, 553, 307

Durant, M., Gandhi, P., Shahbaz, T., Peralta, H. H., \& Dhillon, V. S. 2009, MNRAS, 392, 309

Durant, M., Gandhi, P., Shahbaz, T., et al. 2008, ApJL, 682, L45

Eggleton, P. P. 1983, ApJ, 268, 368

Eikenberry, S. S., Matthews, K., Murphy, T. W., Jr., et al. 1998, ApJL, 506, L31

Esin, A. A., Narayan, R., Cui, W., Grove, J. E., \& Zhang, S.-N. 1998, ApJ, 505,854

Falcke, H., \& Biermann, P. L. 1995, A\&A, 293, 665

Fender, R. P., Belloni, T. M., \& Gallo, E. 2004, MNRAS, 355, 1105

Fitzpatrick, E. L. 1999, PASP, 111, 63

Foight, D., Guver, T., Ozel, F., \& Slane, P. 2015, arXiv:1504.07274

Frank, J., King, A., \& Raine, D. J. 2002, Accretion Power in Astrophysics (Cambridge: Cambridge Univ. Press)

Freudling, W., Romaniello, M., Bramich, D. M., et al. 2013, A\&A, 559, A96

Froning, C. S., Maccarone, T. J., France, K., et al. 2014, ApJ, 780, 48

Gallo, E. 2007, in AIP Conf. Ser. 924, The Multicolored Landscape of Compact Objects and Their Explosive Origins, ed. T. di Salvo et al. (Melville, NY: AIP), 715

Gallo, E., Fender, R. P., \& Pooley, G. G. 2003, MNRAS, 344, 60

Gallo, E., Miller-Jones, J. C. A., Russell, D. M., et al. 2014, MNRAS, 445, 290

Gandhi, P., Blain, A. W., Russell, D. M., et al. 2011, ApJL, 740, L13

Gehrels, N., Chincarini, G., Giommi, P., et al. 2004, ApJ, 611, 1005

Gierliński, M., Done, C., \& Page, K. 2008, MNRAS, 388, 753
Gierliński, M., Done, C., \& Page, K. 2009, MNRAS, 392, 1106

Güver, T., \& Özel, F. 2009, MNRAS, 400, 2050

Hannikainen, D. C., Hunstead, R. W., Campbell-Wilson, D., \& Sood, R. K. 1998, A\&A, 337, 460

Heinz, S., \& Sunyaev, R. A. 2003, MNRAS, 343, L59

Jenniskens, P., \& Desert, F. X 1994, A\&AS, 106, 39

Jimenez-Garate, M. A., Raymond, J. C., \& Liedahl, D. A. 2002, ApJ, 581,1297

La Dous, C. 1989, A\&A, 211, 131

Liu, B. F., \& Meyer-Hofmeister, E. 2001, A\&A, 372, 386

Liu, B. F., Taam, R. E., Meyer-Hofmeister, E., \& Meyer, F. 2007, ApJ, 671,695

Mainzer, A., Bauer, J., Cutri, R. M., et al. 2014, ApJ, 792, 30

Meyer-Hofmeister, E., \& Meyer, F. 2014, A\&A, 562, A142

Mostafa, R., Mendez, M., Hiemstra, B., et al. 2013, MNRAS, 431, 2341

Munari, U., \& Zwitter, T. 1997, A\&A, 318, 269

Narayan, R., \& McClintock, J. E. 2008, NewAR, 51, 733

Narayan, R., \& Yi, I. 1995, ApJ, 452, 710

Neustroev, V. V., Veledina, A., Poutanen, J., et al. 2014, MNRAS, 445, 2424

Palmer, D. M., Barthelmey, S. D., Cummings, J. R., et al. 2005, ATel, 546, 1

Poznanski, D., Prochaska, J. X., \& Bloom, J. S. 2012, MNRAS, 426, 1465

Rahoui, F., Chaty, S., Rodriguez, J., et al. 2010, ApJ, 715, 1191

Rahoui, F., Coriat, M., \& Lee, J. C. 2014, MNRAS, 442, 1610

Rahoui, F., Lee, J. C., Heinz, S., et al. 2011, ApJ, 736, 63

Rahoui, F., Coriat, M., Corbel, S., et al. 2012, MNRAS, 422, 2202

Ratti, E. M., Jonker, P. G., Miller-Jones, J. C. A., et al. 2012, MNRAS, 423, 2656

Reis, R. C., Fabian, A. C., Ross, R. R., \& Miller, J. M. 2009, MNRAS, 395, 1257

Reynolds, M. T., Miller, J. M., Homan, J., \& Miniutti, G. 2010, ApJ, 709, 358

Roming, P. W. A., Kennedy, T. E., Mason, K. O., et al. 2005, SSRv, 120, 95 Russell, D. M., Fender, R. P., Hynes, R. I., et al. 2006, MNRAS, 371, 1334

Russell, D. M., Maitra, D., Dunn, R. J. H., \& Markoff, S. 2010, MNRAS, 405, 1759

Russell, D. M., Markoff, S., Casella, P., et al. 2013, MNRAS, 429, 815

Sault, R. J., Teuben, P. J., \& Wright, M. C. H. 1995, in ASP Conf. Ser. 77, Astronomical Data Analysis Software and Systems IV, ed. R. A. Shaw, H. E. Payne \& J. J. E. Hayes (San Francisco, CA: ASP), 433

Shakura, N. I., \& Syunyaev, R. A. 1973, A\&A, 24, 337

Shaw, A. W., Charles, P. A., Bird, A. J., et al. 2013, MNRAS, 433, 740

Soleri, P., \& Fender, R. 2011, MNRAS, 413, 2269

Soria, R., Wu, K., \& Hunstead, R. W. 2000, ApJ, 539, 445

Titarchuk, L. 1994, ApJ, 434, 570

Tomsick, J. A., Rahoui, F., Kolehmainen, M., et al. 2015, ApJ, 808, 85

Torres, M. A. P., Steeghs, D., Blake, C., et al. 2005, ATel, 566, 1

Vernet, J., Dekker, H., D’Odorico, S., et al. 2011, A\&A, 536, A105

Wilms, J., Allen, A., \& McCray, R. 2000, ApJ, 542, 914

Wilson, W. E., Ferris, R. H., Axtens, P., et al. 2011, MNRAS, 416, 832

Wright, E. L., Eisenhardt, P. R. M., Mainzer, A. K., et al. 2010, AJ, 140,1868

Wu, K., Soria, R., Hunstead, R. W., \& Johnston, H. M. 2001, MNRAS, 320, 177

Zhang, H., Yuan, F., \& Chaty, S. 2010, ApJ, 717, 929

Zurita, C., Durant, M., Torres, M. A. P., et al. 2008, ApJ, 681, 1458 